\title{
SEARCHES FOR NEUTRINOLESS DOUBLE BETA DECAY - RESULTS FROM PHASE I OF THE GERDA EXPERIMENT*
}

\author{
GrZegorz ZuZEL \\ for the GERDA Collaboration \\ The Marian Smoluchowski Institute of Physics, Jagiellonian University \\ Reymonta 4, 30-059 Kraków, Poland \\ (Received October 30, 2013) \\ The result from Phase I of the GERDA experiment is reported. GERDA \\ is carried out at the Gran Sasso Laboratory of INFN in Italy and searches \\ for neutrinoless double beta decay of ${ }^{76} \mathrm{Ge}$. According to the performed \\ analysis, the background index after pulse shape discrimination is about \\ $1 \times 10^{-2} \mathrm{cts} /(\mathrm{keV} \times \mathrm{kg} \times \mathrm{yr})$. No signal was observed and the derived lower \\ limit for the half-life of neutrinoless double beta decay of ${ }^{76} \mathrm{Ge}$ is $\mathrm{T}_{1 / 2}^{0 \nu}>$ \\ $2.1 \times 10^{25}$ yr $(90 \%$ C.L.). The combination with the results from the for- \\ mer Heidelberg-Moscow and IGEx experiments gives an improved limit of \\ $\mathrm{T}_{1 / 2}^{0 \nu}>3.0 \times 10^{25}$ yr $(90 \%$ C.L. $)$. \\ DOI:10.5506/APhysPolB.44.2311
}

PACS numbers: 23.40.-s, 21.10.Tg, 27.50.+e, 29.40.Wk

\section{Introduction}

Thanks to neutrino oscillation experiments, we know that neutrinos are massive particles. Very recent results from reactor experiments have shown that all the mixing angles are different from zero, making the search for $\mathrm{CP}$ violation in the leptonic sector possible. There are, however, very important neutrino properties, which are still to be clarified: the nature of neutrino (Dirac or Majorana), the absolute scale of its mass (from the oscillation experiments only the mass differences can be extracted) and the oscillations with a mass difference of the order of $1 \mathrm{eV}$ (oscillations to sterile neutrinos). One of the possible way to solve the first two issues is to investigate the neutrinoless double beta decay $(0 \nu \beta \beta)$.

* Presented at the XXXVII International Conference of Theoretical Physics "Matter to the Deepest" Ustron, Poland, September 1-6, 2013. 
The double beta decay $(\beta \beta)$ is a second order nuclear process in which a parent nucleus $(A, Z)$ decays to $(A, Z+2)$ with the simultaneous emission of two electrons. Among the possible $\beta \beta$ modes two are of particular interest: the two-neutrino mode $(2 \nu \beta \beta)$ observed already for several isotopes, and the zero-neutrino mode $(0 \nu \beta \beta)$, which violates the lepton number by two units and can occur only if neutrinos are their own antiparticles (Majorana particles). Several possible mechanisms, which could lead to $0 \nu \beta \beta$ are under considerations, i.e. exchange of a light neutrino, right-handed weak currents or exchange of super-symmetric particles. Once it has been observed experimentally, the nature of the process could be further studied, for example, by measuring the energy/angular distribution of the emitted electrons or by comparison of the decay rates of different nuclei. Since in the case of a light neutrino exchange, the $0 \nu \beta \beta$ decay rate is directly proportional to the square of an effective Majorana neutrino mass, the observation of neutrinoless double beta decay will not only tell us about the neutrino nature but also about its absolute mass scale.

The experimental signature of $0 \nu \beta \beta$ decay is a peak at the $Q$ value of the process (for ${ }^{76} \mathrm{Ge}, Q_{\beta \beta}=(2039.061 \pm 0.007) \mathrm{keV}$ [1]). The two most sensitive past experiments, which used ${ }^{76}$ Ge were Heidelberg-Moscow (HDM) [2] and the International Germanium Experiment (IGex) [3, 4]. They found no evidence for the searched peak and set lower limits on the half-life of $T_{1 / 2}^{0 \nu}>$ $1.9 \times 10^{25} \mathrm{yr}$ and $1.6 \times 10^{25} \mathrm{yr}$ at $90 \%$ C.L., respectively. A part of the HDM Collaboration published a claim (not scrutinized until recently) about positive observation (with significance of $4.2 \sigma$ ) of the neutrinoless double beta decay and reported $T_{1 / 2}^{0 \nu}=\left(1.19_{-0.23}^{+0.37}\right) \times 10^{25}$ yr [5]. Later, by applying a pulse shape analysis (PSD), the claim was strengthen to about $6 \sigma$ [6] but because of inconsistencies in the latter, pointed out recently in [7], the comparison of the presented result is restricted to that reported in [5].

Currently, the most sensitive experiments are KamLAND-Zen [8], EXO-200 [9] (both looking for $0 \nu \beta \beta$ decay of ${ }^{136} \mathrm{Xe}$ ), and GERDA [10] employing ${ }^{76} \mathrm{Ge}$. Because the knowledge about the nuclear matrix elements (NME) is needed to relate the different isotopes, the experiments using ${ }^{136} \mathrm{Xe}$ still cannot refute the claim in a model-independent way. This is possible for GERDA since it looks at the same isotope and in Phase I mostly the same detectors as in HDM and IGEX were used.

\section{The GERDA experiment}

The Gerda (Germanium Detector Array) experiment is located at the Laboratori Nazionali del Gran Sasso (LNGS) of INFN in Italy. At the core of the setup, there is an array of p-type high purity germanium detectors (HPGe) operated bare in liquid argon (LAr). LAr is contained in a stain- 
less steel cryostat of $64 \mathrm{~m}^{3}$ volume, which is installed in a water tank instrumented with photomultipliers to detect the Čerenkov light generated by muons (serves as the most outer shield). Cryogenic gas acts both as a coolant (for the HPGe crystals) and as a shield against the external radiation. Initially, the array consisted of eleven semi-coaxial germanium detectors: eight made from enriched germanium (enriched to about $86 \%$ in ${ }^{76} \mathrm{Ge}$ ), and three made from natural germanium, with a total mass of 17.67 and $7.59 \mathrm{~kg}$, respectively. The enriched detectors came from the HDM and IGEX experiments (reused after specific refurbishing processes). Five enriched GERDA Phase II detectors (broad energy germanium - BEGe type manufactured by Canberra [11]) of $3.63 \mathrm{~kg}$ in total were deployed later. The germanium diodes were mounted in low-mass holders and the detector signals were amplified by the custom-made low noise, low radioactivity charge sensitive preamplifiers with $30 \mathrm{MHz}$ bandwidth operated inside LAr. The preamplifiers output pulses were digitized by a 14-bit $100 \mathrm{MHz}$ continuously running ADC (FADC) equipped with anti-aliasing bandwidth filters. In the off-line analysis, the waveforms were digitally processed to reconstruct the event energy. All the construction and operation details of the GERDA experiment can be found in [10].

\section{GERDA Phase I data analysis}

The results discussed here were published in [13-15]. The Phase I data was collected between November 2011 and May 2013. Soon after the deployment, two of the coaxial detectors (called ANG 1 and RG 3) were switched off due to high leakage current. One of the BEGe detectors inserted in July 2012 showed an unstable behavior and it is omitted in the analysis as well. In addition, $5 \%$ of the data was discarded because of temperaturerelated instabilities. Finally, we were operating for 492.3 live days and the total exposure considered for the Phase I $0 \nu \beta \beta$ analysis amounted to 21.6 $\mathrm{kg} \times \mathrm{yr}$ of ${ }^{\mathrm{enr}} \mathrm{Ge}$ detector mass, yielding $(215.2 \pm 7.6)$ mol $\times \mathrm{yr}$ of ${ }^{76} \mathrm{Ge}$ within the active volume. For the analysis, the full data set was subdivided in (a) $17.9 \mathrm{~kg} \times \mathrm{yr}$ exposure of coaxial detectors labeled golden, (b) $1.3 \mathrm{~kg} \times \mathrm{yr}$ exposure of coaxial detectors labeled silver (accumulated during a 30 day period after the insertion of the BEGe detectors resulting in a temporarily higher background index), and (c) $2.4 \mathrm{~kg} \times \mathrm{yr}$ exposure of BEGe detectors labeled BEGe.

The main off-line analysis of the acquired pulses was performed with the GELATIO package [12]. The energy was reconstructed by a digital filter with the semi-Gaussian shaping and its scale was determined with ${ }^{228} \mathrm{Th}$ sources once every one or two weeks. The energy resolutions (FWHM) for coaxial and BEGe crystals measured for the strongest line in the background 
spectrum (1524.6 keV from $\left.{ }^{42} \mathrm{~K}\right)$ was $10 \%$ higher than the resolutions obtained from calibrations (effect of fluctuations of the energy scale between calibrations). The interpolated FWHM for physics data at $Q_{\beta \beta}$ was detector dependent and varied between 4.2 and $5.7 \mathrm{keV}$ for the semi-coaxial detectors, and between 2.6 and $4.0 \mathrm{keV}$ for the BEGe detectors. The exposure-averaged values are $(4.8 \pm 0.2) \mathrm{keV}$ and $(3.2 \pm 0.2) \mathrm{keV}$, respectively.

In order to avoid bias in the event selection criteria, for the first time in the field of the search for $0 \nu \beta \beta$ decay, a blind analysis was performed. The blinding window covered initially the range of $\pm 20 \mathrm{keV}$ around the $Q_{\beta \beta}$ value. After finalization of the energy calibration and the background model the window was reduced to $Q_{\beta \beta} \pm 5(4) \mathrm{keV}$ for the semi-coaxial (BEGe) detectors. Prior to the unblinding, which happened during the collaboration meeting in Dubna in June 2013, the data set to be analyzed, the signal peak fitting method, the background treatment, the pulse shape analysis method and parameters as well as the statistical treatment were fixed and documented such that no further changes were allowed. The background model and the treatments of the background in the $0 \nu \beta \beta$ analysis was published later in [13]. Similarly, the pulse shape analysis methods applied to the coaxial and BEGe data sets were reported in [14].

The achieved background index (after PSD) corresponded to $(11 \pm 2) \times$ $10^{-3} \mathrm{cts} /(\mathrm{keV} \times \mathrm{kg} \times \mathrm{yr})$ for the golden data set, $\left(30_{-9}^{+11}\right) \times 10^{-3} \mathrm{cts} /(\mathrm{keV} \times$ $\mathrm{kg} \times \mathrm{yr})$ for the silver data set, and $\left(30_{-3}^{+4}\right) \times 10^{-3} \mathrm{cts} /(\mathrm{keV} \times \mathrm{kg} \times \mathrm{yr})$ for the BEGe data set. A $0 \nu \beta \beta$ signal acceptance was $\epsilon^{\mathrm{PSD}}=\left(0.90_{-0.09}^{+0.05}\right)$ and $\epsilon^{\mathrm{PSD}}=(0.92 \pm 0.02)$ for the coaxial and for the BEGe detectors, respectively.

After unblinding the GERDA data showed no indication of a peak at $Q_{\beta \beta}$ - see Fig. 1. To derive the signal strength $N^{0 \nu}$ and a frequentist coverage interval, a profile likelihood fit of the three data sets was performed. The best fit delivered $N^{0 \nu}=0$, what means no excess of events in the peak region above the background. The limit derived by GERDA for the half-life of ${ }^{76} \mathrm{Ge}$ with respect to neutrinoless double beta decay is [15]

$$
T_{1 / 2}^{0 \nu}>2.1 \times 10^{25} \text { yr } \quad(90 \% \text { C.L. }) .
$$

The limit on the half-life corresponds to $N^{0 \nu}<3.5$ counts (solid/blue curve in Fig. 1). Assuming that the claim [5] is correct, GerDA should observe $(5.9 \pm 1.4)$ decays in the peak over $(2.0 \pm 0.3)$ background events in the $\pm 2 \sigma$ window after the PSD cuts (dashed/red curve in Fig. 1). The probability for a downward fluctuation of the signal, which provides $N^{0 \nu}=0$ counts as the best fit would be only $1 \%$. The profile likelihood fit was also extended to include the spectra from HDM and IGEX, and in such a case the best fit yields again $N^{0 \nu}=0$ and a limit of

$$
T_{1 / 2}^{0 \nu}>3.0 \times 10^{25} \text { yr } \quad(90 \% \text { C.L. }) .
$$



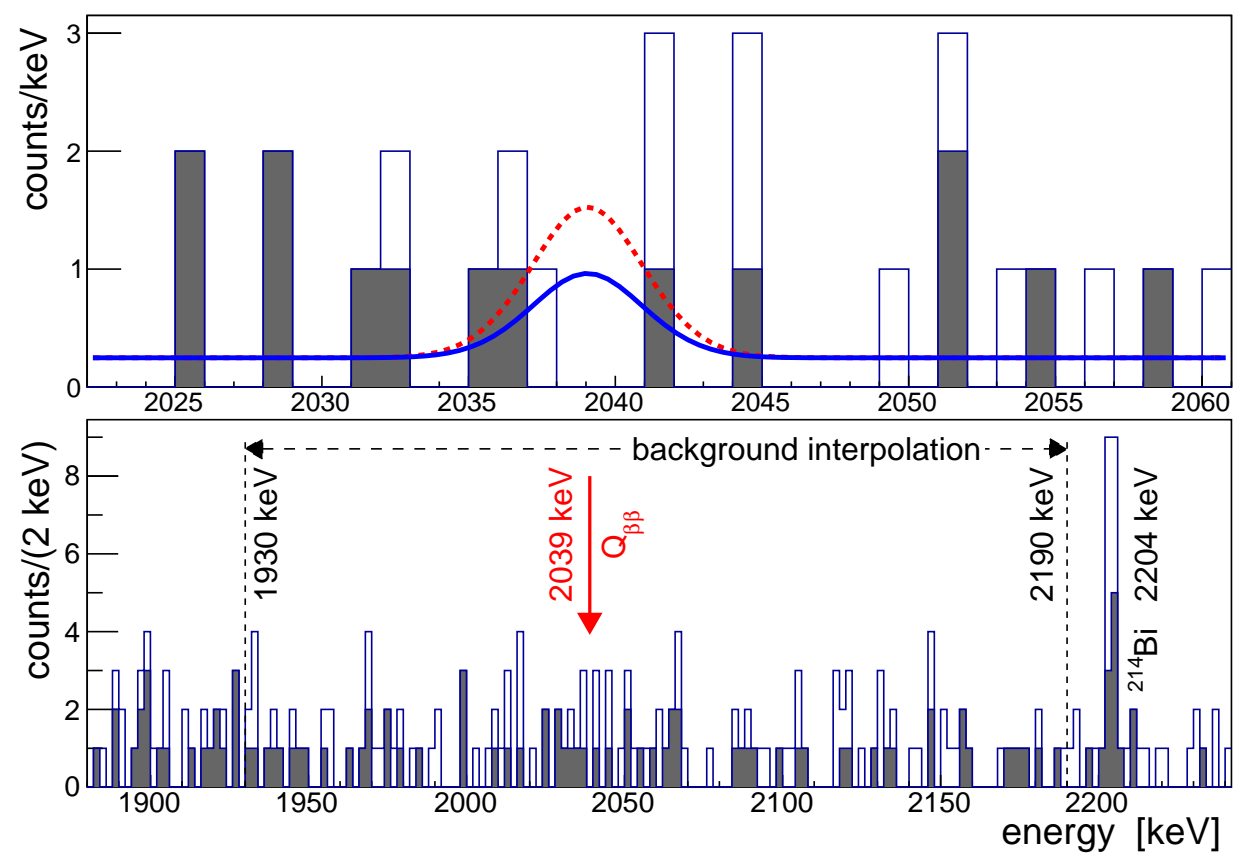

Fig. 1. The GERDA Phase I energy spectrum from all enriched detectors with and without PSD shown by the filled and open histograms, respectively. The lower panel shows the region used for the background interpolation. The spectrum in the upper plot is zoomed to $Q_{\beta \beta}$ and it was superimposed with the expectations (with PSD selection) based on the central value of [5] $\left(T_{1 / 2}^{0 \nu}=1.19 \times 10^{25} \mathrm{yr}\right.$ - dashed $\left./ \mathrm{red}\right)$ and with the $90 \%$ upper limit derived by GERDA $\left(T_{1 / 2}^{0 \nu}=2.10 \times 10^{25} \mathrm{yr}\right)$.

\section{Towards GERDA Phase II}

In Phase II, beside the increase of the active mass by about $20 \mathrm{~kg}$ (30 BEGe detectors), the main goal is to further reduce the background by one order of magnitude. This will be realized by using less material but of higher radio-purity in the vicinity of the diodes (i.e. new silicon holders, each accommodating two BEGe diodes, will be used) and by applying an active veto ( $\mathrm{LAr}$ will be instrumented).

All new BEGe detectors have been fully characterized, transported to LNGS and are ready to be used. The tests of new detector holders with veryfront end electronics (also re-designed for Phase II) and LAr veto system are ongoing. The new lock system allowing for handling of appropriately larger number of detectors will be installed at the end of 2013. The commissioning of Phase II will start subsequently. 


\section{Conclusions}

In Phase I of GERDA the assumed (in the proposal) experimental conditions (i.e. background index, exposure) were achieved. The obtained lower limit for the neutrinoless double beta decay is the strongest up to date. By using the ${ }^{76} \mathrm{Ge}$ isotope, GERDA can also test the claim [5] in a fully modelindependent way and the presented Phase I result strongly disfavors it. This finding is even stronger if available data from the former IGEX and HDM experiments are taken into account in the combined analysis. The calculated range for the upper limit on the effective electron neutrino mass $m_{\beta \beta}$ is $0.2-0.4 \mathrm{eV}$. A detailed description of the GERDA data evaluation criteria and procedures can be found in [13-15].

The participation of the group from the Jagiellonian University in the GERDA experiment is supported by the Polish National Science Centre, grant No. DEC-2011/01/M/ST2/00755.

\section{REFERENCES}

[1] B.J. Mount, M. Redshaw, E.G. Myers, Phys. Rev. C81, 032501 (2010).

[2] H.V. Klapdor-Kleingrothaus et al. [Heidelberg-Moscow Collaboration], Eur. Phys. J. A12, 147 (2001).

[3] C.E. Aalseth et al. [IGEX Collaboration], Phys. Rev. D65, 092007 (2002).

[4] C.E. Aalseth et al. [IGEX Collaboration], Phys. Rev. D70, 078302 (2004).

[5] H.V. Klapdor-Kleingrothaus, I.V. Krivosheina, A. Dietz, O. Chkvorets, Phys. Lett. B586, 198 (2004).

[6] H.V. Klapdor-Kleingrothaus, I.V. Krivosheina, Mod. Phys. Lett. A21, 1547 (2006).

[7] B. Schwingenheuer, Ann. Phys. (Berlin) 525, 269 (2013).

[8] A. Gando et al., Phys. Rev. Lett. 110, 062502 (2013).

[9] M. Auger et al., Phys. Rev. Lett. 109, 032505 (2012).

[10] K.-H. Ackermann et al. [GERDA Collaboration], Eur. Phys. J. C73, 2330 (2013).

[11] Canberra Semiconductor NV, Lammerdries 25, B-2439 Olen, Belgium.

[12] M. Agostini, L. Pandola, P. Zavarise, O. Volynets, JINST 6, P08013 (2011).

[13] M. Agostini et al. [GERDA Collaboration], under review at Eur. Phys. J. C, arXiv:1306.5084 [physics.ins-det].

[14] M. Agostini et al. [GERDA Collaboration], Eur. Phys. J. C73, 2583 (2013).

[15] M. Agostini et al. [GERDA Collaboration], Phys. Rev. Lett. 111, 122503 (2013). 